\title{
LE PLURILINGUISME TEXTUEL ET LES MECANISMES DE SA MISE EN GEURE CHEZ AHMADOU KOUROUMA ET PATRICK CHAMOISEAU
}

\section{El hadji CAMARA, Université Western Ontario}

Parler du plurilinguisme textuel dans le roman francophone, c'est poser la question du choix de la langue d'écriture chez l'écrivain francophone, mais aussi c'est évoquer à la fois les éléments constitutifs qui participent de la définition même de la littérature francophone. Par définition, un écrivain francophone, c'est celui qui écrit en français, peut-on dire de manière tautologique. Mais cette définition passe sous silence la dimension institutionnelle voire idéologique à l'œuvre dans la constitution de tout groupe social.

Selon Dominique Combe, les écrivains francophones forment un groupe dans lequel des hommes et des femmes, au delà la langue d'écriture qu'ils ont en commun, « expriment le sentiment d'appartenir, à travers la langue française, à une communauté de pensée, de culture » (14). Il souligne ainsi le fait que le projet littéraire en tant que tel engage au plus haut point « la personnalité de l'écrivain » en permettant de l'identifier, le texte étant une lecture possible du monde parmi tant d'autres.

Cependant, il faut reconnaître que tout texte possède sa propre identité, par ses langues et ses référents extratextuels, que ceux-ci soient ceux de l'auteur ou non. Dès lors, comment distinguer la langue de l'auteur de celle du texte ? Et dans quelles mesures les mécanismes de la mise en texte des langues concourent-ils à la production du sens dans l'œuvre?

Alors qu'Ahmadou Kourouma se vante de faire parler ses personnages dans la langue de leur milieu social et culturel, Patrick Chamoiseau, se qualifiant lui-même de marqueur de parole, affirme retranscrire les paroles du conteur. Dès lors, à travers une analyse comparative, il sera question d'analyser d'une part les éléments qui permettent de définir un texte francophone de manière générale, et d'autre part de voir comment la textualisation des langues est mise en pratique chez ces deux écrivains francophones. 
Nous privilégierons l'approche sociocritique dans la mesure où les auteurs étudiés ici revendiquent eux-mêmes l'encrage de leurs textes dans les contextes sociopolitiques, historiques et culturels qui les ont vus naître. Cela nous permettra surtout de voir comment le contexte éclaire ces œuvres et comment, à partir d'elles, nous pourrions avoir une meilleure connaissance dudit contexte.

\section{Les éléments constitutifs du texte francophone}

Pour Michel Beniamino, dans La francophonie littéraire : essai pour une théorie (1987), parler de «littérature francophone », c'est parler d'une littérature qui se déploie sur un vaste espace géographique disparate, avec des variétés de français qui résultent d'un environnement institutionnel et d'un contexte socio-historique particulier. La prise en compte de ces facteurs permet une meilleure compréhension des textes.

Dans Le rôle des revues littéraires et des maisons d'édition dans la spécification de la (des) littérature(s) de l'Afrique subsaharienne francophone (1991), à travers une approche sociohistorique, Josias Semujanga souligne le rôle capital de l'institution littéraire dans la spécification du champ littéraire africain francophone. Voilà en quelques mots la preuve, si besoin en est, qu'en plus de la dimension linguistique, l'aspect institutionnel constitue l'un des éléments de définition de la littérature francophone en ce sens qu'il pourrait prédéterminer l'horizon d'attente.

\section{La langue d'écriture et l'horizon d'attente}

Le choix d'une langue d'écriture n'est jamais anodin comme le montrent ces propos de Lise Gauvin dans Les langues du roman. Du plurilinguisme comme stratégie textuelle (1999) :

La problématique des interactions langues / littératures est complexe et met en cause aussi bien des enjeux institutionnels - l'autonomie d'une littérature, les conditions de son émergence, la relation qui s'y établit entre l'écrivain et le public -, politiques - le statut d'une langue et de ceux qui la parlent -, que proprement littéraires, à savoir quels sont les modèles dont dispose l'écrivain pour représenter les rapports sociaux entre les langues ou les niveaux de langue. (53) 
Il y a deux enseignements majeurs à retenir dans ces propos : l'interaction des langues et la représentation des rapports sociaux entres les langues. Il s'y ajoute, dans le contexte des littératures issues d'ex-colonies françaises, que ces problématiques sont souvent liées à celle de l'identité, l'identité de l'écrivain en particulier. Et pendant longtemps, la question du public à qui s'adresse l'écrivain francophone a alimenté les débats.

Parmi d'autres auteurs, Albert Memmi pensaient qu'il était condamné d'avance à ne s'adresser qu'à un seul public, celui de l'ex-colonisateur. Dans Le Portrait du colonisé (1957), Memmi envisageait même la mort de la littérature du colonisé, puisque les perspectives qui s'offraient à lui étaient des impasses. Soit, il y aura tarissement naturel de la littérature du colonisé, disait-il, parce que les générations futures décideront spontanément d'écrire dans leur langue maternelle qu'elles auront trouvée après la décolonisation, mais du fait que cette langue n'est pas écrite, elles manqueront de lecteurs. Soit, ces auteurs décideront de se fondre dans la littérature métropolitaine, et dans ce cas, ce sera un suicide, car ils n'existeront même pas. En tout état de cause, dira Memmi « la littérature colonisée de langue européenne semble condamnée à mourir jeune $\gg(105)$.

Or, tout indique que ces littératures se sont développées à un rythme insoupçonné et se sont plus ou moins autonomisées. Quant à savoir pourquoi ils écrivent en français, des auteurs comme Édouard Glissant, Patrick Chamoiseau ou Ahmadou Kourouma ont répondu à cette interpellation de fort belle manière. Pour Chamoiseau par exemple, il est hors de question de s'automutiler en renonçant à écrire en français ou en choisissant d'écrire uniquement en créole. Catherine Détrie note dans Figures de l'interculturalité (1996) à propos de ce choix cornélien de la part des écrivains antillais :

Si la plupart des Antillais sont créolophones, très peu sont créolographes (...). Choisir le créole c'est : - se cantonner à un lectorat réduit, s'interdire les échanges et confrontations fructueux entre soi-même et un lectorat à l'existence problématique. De plus c'est aussi se couper de la part de l'autre en soi, donc s'amputer d'une partie de soi-même - choisir de ne pas se confronter à la littérature occidentale, s'affirmer radicalement autre. Or la première (et même quelquefois la seule) littérature à laquelle l'enfant antillais est confronté est la littérature occidentale : l'identité antillaise passe par cette appréhension littéraire. (110) 
La solution toute trouvée par les auteurs antillais comme Chamoiseau ou Glissant, c'est de s'approprier le français pour qu'il soit habité par une conscience créole authentique. Cela permet surtout l'inscription de la tradition et des formes orales discursives dans la production littéraire antillaise, alors que pour Kourouma, «Les Africains, ayant adopté le français, doivent maintenant l'adapter et le changer pour s'y trouver à l'aise [...]» (Dumont 115). C'est la prise en compte de tels impératifs qui entraîne la cohabitation de plusieurs univers linguistiques dans un texte francophone. L'œuvre romanesque de Kourouma en est une parfaite illustration.

\section{Les langues du texte ou le plurilinguisme textuel}

La présence de différentes langues ou de différents niveaux de langue dans les œuvres littéraires francophones n'est pas un phénomène nouveau. Cependant, le but était souvent de faire correspondre les personnages avec leur milieu social, ethnique ou culturel. La manière de s'exprimer d'un personnage pouvait donner des indications sur son environnement. Mais la textualisation des langues, en tant qu'objet littéraire au point de concurrencer des thèmes classiques (engagement, procès du colonialisme, portait...) est assez récente. Qualifiant ce phénomène d'hétérolinguisme, Rainier Grutman le définit dans Des langues qui résonnent. L’hétérolinguisme au XIXe siècle québécois comme étant « la présence dans un texte d'idiomes étrangers, sous quelque forme que ce soit, aussi bien que de variétés (sociales, régionales ou chronologiques) de la langue principale »(Grutman 37). Alors qu'est-ce qui motive la pratique de ce plurilinguisme dans les textes?

A notre avis, c'est la situation même de l'écrivain francophone, côtoyant plusieurs univers linguistiques, qui l'expose au phénomène de plurilinguisme textuel. Nous partons du principe que l'œuvre littéraire quelle qu'elle soit, porte en elle les marques de son environnement (contexte social, politique et culturel). Gauvain reconnaît l'influence de l'environnement sur l'écrivain en affirmant :

Dans un pareil contexte, la situation des écrivains francophones est emblématique d'un parcours qui les condamne, de quelque lieu qu'ils proviennent, à penser la langue. La proximité des autres langues, la situation de diglossie sociale dans laquelle ils se trouvent le plus souvent immergés, une première déterritorialisation constituée par le passage de 
l'oral à l'écrit, et une autre, plus insidieuse, créée par des publics immédiats ou éloignés, séparés par des acquis culturels et langagiers différents, sont autant de faits qui les obligent à énoncer des stratégies de détours. (10)

Au-delà de ces stratégies d'écriture qui se manifestent par les emprunts, l'intégration de mots étrangers ou la transposition de la structure d'une langue dans une autre, la remarque de Gauvin met en exergue l'impossibilité pour un écrivain francophone de se départir de son univers socioculturel et linguistique. Cette dépendance de l'écrivain à l'environnement s'explique notamment selon Abdelkebir Khatibi dans l'essai $D u$ Bilinguisme (1985) par le fait qu'il y a toujours une interconnexion entre la langue maternelle et la langue étrangère, surtout si cette dernière est la langue d'écriture pour l'auteur: «La langue 'maternelle' est à l'œuvre dans la langue étrangère. De l'un à l'autre se déroulent une traduction permanente et un entretien en abyme [...] (171). La prise en compte effective de ce plurilinguisme entraîne la cohabitation de plusieurs univers linguistiques dans un même texte. Pour Jean-Marc Moura aussi, soulignant le rôle de passeur de langue de l'écrivain francophone, « la conscience linguistique, développée chez tout écrivain, est, en effet, cardinale dans un contexte plurilingue » (94). C'est la raison pour laquelle, il s'avère nécessaire d'analyser les voies par lesquelles passe la multiplication des langues dans un texte francophone.

\section{Les mécanismes de mise en œuvre du plurilinguisme dans les textes}

Dans la préface de son livre Des langues qui résonnent. L'hétérolinguisme au XIXe siècle québécois, Grutman constate:

Un texte littéraire est rarement uniforme au point de vue de la langue. Plus souvent qu'on ne le croirait, il est entrelardé d'éléments hétérogènes. En plus d'intégrer plusieurs niveaux et diverses strates historiques de son idiome principal, il fait une place plus ou moins large à d'autres langues: cela peut aller du simple emprunt lexical aux dialogues en parlers imaginaires, en passant par les citations d'auteurs étrangers. (11)

Alors comment se manifeste cette textualisation des langues chez Kourouma et chez Chamoiseau? 


\section{La transposition comme mécanisme de représentation chez Kourouma}

Nous empruntons ce terme de «transposition» à Laté Lawson-Héllu, qui le définit comme étant : «le processus d'expression du contenu énonciatif d'une langue d'origine, langue-source, dans une langue d'arrivée, langue-cible » (7).

A la lecture des œuvres de Kourouma, nous pouvons déduire qu'il est question d'un tel processus. En effet, on a souvent analysé la question linguistique chez Kourouma comme un mécanisme d'appropriation de la langue française qui confond les structures du français à celles du malinké. A ce titre, les études consacrées aux Soleils des indépendances (1970) sont de parfaits exemples. Comme le dit Edmond Biloa, «Dans ce roman, Kourouma mélange allégrement le discours français à la parole malinké pour produire un métissage linguistique truculent. La bizarrerie prend forme dès le début du roman par la première phrase - 'Il y avait une semaine qu'avait fini dans la capitale Koné Ibrahim...' » (np).

De toute évidence, il y a un dysfonctionnement de la langue française, mais il ne s'agit ni de métissage linguistique, ni de bizarrerie à notre avis. Puisque Kourouma n'a procédé à aucune alternance codique, ni même d'intégration de mots malinké dans ses propos. Il est question ici plutôt de transposition qui permet à l'auteur de faire l'annonce du décès d'Ibrahim en gardant textuellement les subtilités du malinké en la matière, en livrant un message bien codifié.

Car l'auteur avait le choix entre plusieurs verbes ou expressions pour faire cette annonce. En effet, en malinké, les verbes et expressions pouvant dire «mourir » sont nombreux, il s'agit entre autres de «n'être plus », « être parti », « s'être couché », «s'être reposé », etc. En portant son choix sur «finir », l'auteur voudrait simplement dire que Koné Ibrahim n'a laissé derrière lui aucun héritier, que la branche le concernant dans un éventuel arbre généalogique s'arrête complètement avec sa mort.

$\mathrm{Au}$ contraire, dire de quelqu'un après sa mort qu'il «s'est couché » «ou s'est reposé' », c'est souligner le caractère apaisant de la mort par rapport à une éventuelle douleur qu'il subirait en étant en vie, alors qu'on dirait de quelqu'un qui a laissé un vide sur le plan affectif après sa mort qu'il « est parti ». Connaissant toutes ces nuances de la langue d'origine, l'auteur ne pouvait procéder que par transposition. Car, comme le dit Lawson-Héllu, «Dans le cas de la transposition, le résultat visible de l'opération est à 
même de maintenir dans la langue d'arrivée toutes les charges sociales, identitaires voire normatives et symboliques de la langue d'origine » (8). Ainsi, le verbe « finir », comme euphémisme pour désigner la mort, prend tout son sens en se référant à un contexte socioculturel bien précis.

Une autre occurrence de cette transposition est à noter dans les propos du narrateur expliquant les raisons profondes de la chute de Fama Doumbouya dans Les Soleils des Indépendances:

On l'avait bien prévenu. Les gens de l'indépendance ne connaissent ni la vérité, ni l'honneur, ils sont capables de tout, même fermer l'œil sur une abeille. On lui avait dit que là où les graterons percent la coque des œufs de pintade, ce n'est pas un lieu où le mouton à laine peut aller. Il s'est engagé, il a voulu terrasser les soleils des Indépendances, il a été vaincu. Il ne ressemblait maintenant qu'à une hyène tombée dans un puits ; il ne lui restait à attendre que de la volonté d'Allah; que de la volonté de la mort. (Kourouma 168).

Pour percer la signification de ces propos, il est nécessaire de comprendre le rapport qu'entretiennent les gens de Horodougou réputés pour leur bravoure et l'univers animalier évoqué ici par quelques bêtes symboliques à savoir l'hyène, le mouton et la pintade. Si le mouton a toujours été un animal bien perçu, l'hyène représente tout ce qui est négatif dans la conscience collective malinké. Ainsi, comparer quelqu'un à une hyène enfermée dans un puits, c’est souligner sa déchéance totale. Fama Doumbouya, issue d'une famille princière, se trouve au plus bas de l'échelle sociale par la faute des Soleils des Indépendances. Mais en même temps, le courage de ceux qui sont capables de fermer l'œil sur une abeille, dépasse celui des gens de Horodougou. La comparaison dans ce contexte, même si elle met en lumière le triste sort de Fama Doumbouya, l'exonère dans la mesure où il ne pouvait rien faire face aux nouvelles autorités. On voit bien que Kourouma ne pouvait faire autrement que de recourir à la transposition s'il ne voulait pas perdre l'image si évocatrice qui transparaît dans ses propos.

Ce procédé de transposition, qui permet l'interconnexion de plusieurs langues voire plusieurs discours, est très fréquent chez Kourouma; il pourrait même être un leitmotiv en regardant de près son œuvre romanesque. Lorsque le narrateur d'Allah n'est pas obligé (2000) dit, dès le début du roman, son attachement aux dictionnaires pour trouver les gros mots et le sens des africanismes, nous pourrions en déduire que Kourouma lui-même 
procède de cette manière. Mais, à y regarder de près, nous pouvons affirmer que la raison principale du recours à la transposition permettant la cohabitation de plusieurs univers linguistiques dans un même texte s'explique par l'impossibilité de tout traduire textuellement. A cet effet, l'épigraphe de son roman Monnè, outrages et défis (1998) est assez révélatrice : «Un jour le Centenaire demanda au Blanc comment s'entendait en français le mot monnè. 'Outrages, défis, mépris, injures, humiliations, colère rageuse, tous ces mots à la fois sans qu'aucun le traduise véritablement', répondit le Toubab qui ajouta : 'En vérité, il n'y a pas chez nous, Européens, une parole rendant totalement le monnè malinké' » (9). Ainsi, afin de rendre le plus fidèlement possible toute l'idée qu'il y a derrière ce mot monnè, Kourouma le juxtapose avec outrages et défis dans le titre de son roman.

Khatibi explique cette difficulté de traduction de manière éloquente : « Ce serait une sorte de folie de croire qu'une langue (n'importe laquelle), puisse écrire et récrire une autre langue de l'intérieur, et puisse la domestiquer selon une loi parfaitement invisible. Pourtant, un tel désir de transposition radicale, de traversée renversante d'une langue à l'autre, transite dans certains textes tentés par l'intraduisible » (192). Il est donc impossible pour Kourouma de traduire le malinké dans toutes ses subtilités sans le trahir.

\section{L'intégration des mots et des structures du créole chez Chamoiseau}

Comme chez tout écrivain en situation multiculturelle, la problématique de la langue est constamment au cœur des œuvres de Chamoiseau, surtout dans Solibo Magnifique (1988), Texaco (1992) et Écrire en pays dominé (1997). Quand bien même, il refuse l'artifice d'un créole uniformisé et d'un français figé ; il ne cesse de faire cohabiter en situation diglossique les deux langues. Selon Détrie, « La situation de diglossie (héritage colonial, donnée sociohistorique) est toujours en travail et ne peut être gommée, la diglossie étant vécue comme une tension conflictuelle entre le même et l'autre » (104).

Cependant, à défaut d'être gommées, les tensions résultant de la diglossie sont résolues par la langue de la narration, laquelle est décrite par Détrie comme «la représentation d'un dialogisme interlectal qui refuse le modèle du sujet diglossique auquel il substitue le sujet de la diglossie, la diglossie étant alors envisagée non plus comme un clivage sclérosant, mais comme une dynamique structurante »(105). De ce 
fait, on assiste à la naissance d'une langue tierce, celle du texte qui réconcilie les deux pôles fondateurs de l'identité linguistique des Antilles à savoir le créole et le français. Le choix d'une telle langue est motivé par le lectorat visé et par le souci de rendre compte d'un état d'être.

Ainsi, dans ses œuvres, Chamoiseau mettra en pratique cette esthétique pour explorer les différentes facettes de la société antillaise. Que ce soit dans Texaco ou dans Écrire en pays dominé, l'auteur adopte un positionnement assez particulier vis-à-vis des formes narratives dominantes en y associant les formes orales liées à sa culture : « La langue française sera à tout moment intégrée, moquée, et déconstruite sous des modes erratiques » (173). Chamoiseau prône clairement une nouvelle forme d'écriture. Il privilégie surtout une démarche scripturale qui permet de rendre compte du quotidien antillais, à l'image de l'Informatrice dans Texaco: «Elle mélangeait le créole et le français, le mot vulgaire, le mot précieux, le mot oublié, le mot nouveau..., comme si à tout moment elle mobilisait (ou récapitulait) ses langues. Elle avait des périodes de voixpas-claire comme certains grands conteurs »(424). C'est ce qui explique la fréquence dans ses textes des marques du créole et des spécificités culturelles qu'un lecteur étranger aura du mal à comprendre parfaitement sans annotation.

Mais faut-il le signaler, Chamoiseau ne met pas en cause la littérature européenne en tant que forme apte à dire un imaginaire occidental par des marques propres à la pensée et à la langue françaises. Cependant, il conteste la pertinence de la même langue et de la même pensée pour rendre compte d'un réel sans rapport avec le réel occidental, c'est-à-dire le réel antillais. Dans Écrire en pays dominé, à propos de la langue française, il affirme sans ambages : «La langue qui ne sait pas quoi mettre dans la bouche des nègres-de-terre, et qui assiège mal les événements de leur esprit » (79). L'auteur met en cause l'inadéquation de la langue du dominateur pour dire l'univers du dominé. Glissant, quant à lui, tout en rejetant la vision occidentale du monde, masquée sous le couvert de l'universalisme, propose une ouverture beaucoup plus grande, prenant en compte ce qu'il appelle « la créolisation» du monde où toutes les langues entrent en dialogue et s'enrichissent mutuellement. 


\section{Conclusion}

En somme, si la langue française est un des éléments qui participent de la définition de la littérature francophone, la conscience chez l'écrivain de l'existence d'une variété d'idiomes en contact donne une clé pour une lecture avisée des œuvres. Dès lors, tout effort de définition du champ littéraire francophone doit prendre en compte la problématique linguistique comme élément constitutif du roman francophone.

Mais le plurilinguisme textuel fait appel lui-même à plusieurs aspects dans l'analyse des textes, car, au-delà de la présence d'une ou des langues dans le texte à travers divers mécanismes mis en pratique, il nous semble que c'est l'environnement (le contexte institutionnel, idéologique, culturel, social, politique, etc.) qui guide toute production littéraire.

Pour Chamoiseau, l'écrivain antillais doit faire naître un nouveau langage capable de dire l'être créole, ses douleurs comme ses aspirations à un monde juste et sans domination des uns par les autres. C'est à ce titre seulement que la littérature antillaise prendra son envol, car dira-t-il, « comment écrire alors que ton imagination s'abreuve, du matin jusqu'aux rêves, à des images, des pensées, des valeurs qui ne sont pas les tiennes ? Comment écrire quand ce que tu es végète en dehors des élans qui déterminent ta vie ?» (Écrire en pays, 17).

Quant à Kourouma, ses œuvres sont de très belles illustrations de la cohabitation de différents univers linguistiques. Mais la langue de Kourouma, pour reprendre le terme de Makhily Gassama, ce n'est pas seulement la juxtaposition du français et du malinké, c'est aussi et surtout une langue qui parvient à traduire les pensées profondes de ses personnages dans une langue qui n'est pas la leur.

\section{Ouvrages cités}

Beniamino, Michel. La francophonie littéraire: essai pour une théorie. Paris: L'Harmattan, 1999.

Biloa, Edmond. «Appropriation, déconstruction du français et insécurité linguistique dans la littérature africaine d'expression française ». Appropriation de la langue française dans les littératures francophones. De l'Afrique subsaharienne du 
Maghreb et de l'Océan indien. Dir. Moussa Daff. Sylvains les Moulins : Synergies Revue du Gerflint 2 (2002).

Bisanswa, K. Justin et Tetu, Michel. Francophonie au pluriel. Québec : CIDEF-AFI, 2003

Bres, Jacques, et Siblot, Paul. Figures de l'interculturalité. Montpellier : PU Paul Valéry, Collection Praxiling, 1996.

Chamoiseau, Patrick. Solibo Magnifique. Paris : Gallimard, 1988.

---. Texaco. Paris : Gallimard, 1992.

---. Écrire en pays dominé. Paris : Gallimard, 1997.

Combe, Dominique. Poétiques francophones. Paris : Hachette, 1995.

Daff, Moussa, Dir. Appropriation de la langue française dans les littératures francophones. De l'Afrique subsaharienne du Maghreb et de l'Océan indien. Sylvains les Moulins : Synergies Revue du Gerflint 2 (2002).

Dumont, Pierre. L'interculturel dans l'espace francophone. Paris : L'Harmattan, 2001.

Gassama, Makhily. La langue d'Ahmadou Kourouma ou le français sous le soleil d'Afrique. Paris : Broché, 1995.

Gauvin, Lise. Les langues du roman. Du plurilinguisme comme stratégie textuelle. Montréal : PU de Montréal, 1999.

Glissant, Edouard. Traité du Tout-Monde. (Poétique IV). Paris : Gallimard, 1997.

---. Poétique de la Relation. (Poétique III). Paris : Gallimard, 1990.

Grutman, Rainier. Des langues qui résonnent. L'hétérolinguisme au $19^{e}$ siècle québécois. Québec : Fides-Cétuq, 1997.

Khatibi, Abdelkebir. Du Bilinguisme. Paris : Denoël, 1985.

Kourouma, Ahmadou. Allah n'est pas obligé. Paris : Seuil, 2000.

---. Monnè, outrages et défis. Paris : Seuil, 1998.

---. Les Soleils des indépendances. Paris : Seuil, 1970.

Lawson-Hellu, Laté. «Textualité et transposition hétérolinguistique dans le roman francophone : Pour une théorie générale du plurilinguisme littéraire ». Conférence présentée au Colloque international «La Traversée dans le roman francophone » de l’Université Laval, Québec, juin 2005. (à paraître) 
Memmi, Albert. Portrait du colonisé, précédé du portrait du colonisateur. 1957. Paris : Gallimard, 1985.

Moura, Jean-Marc. «La francophonie littéraire : quelle diversité et quelle cohérence ?» Francophonie au pluriel. Dirs. K. Justin Bisanswa et Tétu, Michel. Québec : CIDEF-AFI, 2003. 93-98.

Semujanga, Josias. «Le rôle des revues littéraires et des maisons d'édition dans la spécification de la (des) littérature(s) de l'Afrique subsaharienne francophone ». Revue Etudes littéraires 24 : 2 (1991) : 99-112. 